PDFlib PLOP: PDF Linearization, Optimization, Protection

Page inserted by evaluation version www.pdflib.com - sales@pdflib.com 


\title{
An orthotopic floor-of-mouth model for locoregional growth and spread of human squamous cell carcinoma
}

\author{
B. Henson ${ }^{1 *}$, F. Li $^{1 *}$, D. D. Coatney ${ }^{1}$, T. E. Carey ${ }^{1,2}$, R. S. Mitra ${ }^{1}$, K. L. Kirkwood ${ }^{1}$, N. J. D’Silva ${ }^{1,3}$ \\ ${ }^{1}$ Department of Periodontics and Oral Medicine, University of Michigan School of Dentistry; ${ }^{2}$ Department of Otolaryngo- \\ logy; ${ }^{3}$ Department of Pathology, University of Michigan Medical School, Ann Arbor, MI, USA
}

\begin{abstract}
The molecular investigation of head and neck cancer targets requires the utilization and optimization of established animal models to characterize the effects of gene transcription and protein expression on invasion and metastasis. Floor-of-the-mouth murine models have been developed to study tumor growth, invasion, and metastasis of murine squamous cell carcinoma (SCC) cells in immunocompetent mice and invasion and metastasis of human SCC cells in nude mice. However, there are tumor cell lines that do not produce tumors in mice, using standard techniques, thus reducing the utility of the model to study specific genetic or treatment conditions. Furthermore, these techniques require large tumor volumes raising the possibility of airway compromise. In this report, we detail significant modifications to the orthotopic floor-of-mouth murine model for human SCC to facilitate predictable growth of a large panel of University of Michigan SCC cell lines. Furthermore, we describe the use of bioluminescence and micro-computed tomography to monitor tumor growth and bony invasion.

J Oral Pathol Med (2007) 36: 363-70
\end{abstract}

Keywords: murine; orthotopic; SCC

\section{Introduction}

Head and neck squamous cell carcinoma (HNSCC) has a survival rate of $<50 \%$, a prognosis that is poorer than breast or colon cancer or melanoma (1). This poor prognosis is due to two confounding issues: late detection of HNSCC and inadequate treatment options for advanced disease (2). Identification of the molecular events that regulate the pathogenesis, progression, and

Correspondence: NJ D'Silva, 1011 North University Avenue, Room 5217, Department of Periodontics and Oral Medicine, University of Michigan School of Dentistry, Ann Arbor, Michigan, 48109-1078, USA. Tel: + 1734764 1543, Fax: +1 734764 2469, E-mail: njdsilva@umich.edu

*These authors contributed equally to this manuscript.

Accepted for publication February 14, 2007 treatment response of HNSCC will provide objective markers and specific treatment targets, thereby reducing the morbidity of the disease. The molecular investigation of a disease process is greatly enhanced by reliable animal models that replicate the human disease. In this regard, murine models for oral cancer have facilitated investigation of HNSCC (3-5). Xenograft tumor models involve injection and growth of tumor cells in the subcutaneous tissue of the hind leg or nape of mice (6). However, these models do not replicate the locoregional biology of the tumor, an important element of human HNSCC, which grows in proximity to abundant vasculature and bone. These drawbacks were addressed by orthotopic, floorof-mouth murine models which facilitate studies on tumor growth, invasion, and metastasis of murine squamous cell carcinoma (SCC) cells in immunocompetent mice (3) and invasion and metastasis of human SCC cells in immunodeficient mice $(4,5,7)$. However, we observed that the model is effective only for specific HNSCC cell lines. A robust model is essential to study multiple HNSCC cell lines that may represent different genetic mutations, thereby facilitating pre-clinical studies that are relevant to multiple HNSCCs. Low tumor injection volume and tumor cell dose minimize the potential for airway compromise and facilitate longterm tumor growth in vivo, respectively. Hence, the murine model for human HNSCC was modified and optimized to address these problems. Using a matrigel basement membrane matrix in this model, tumor induction is successful even with challenging HNSCC cell lines; the incidence of injection-induced mortalities is low, and in the initial stages of tumor development, tumor cells are restricted to a small area, thereby reducing tumor cell dose. The prolonged duration of tumor growth from this small cell innoculum allows development of metastases. Furthermore, SCC cells were labeled with a luciferase reporter and tumor growth and bone invasion were assayed by bioluminescence and micro-computed tomography $(\mu \mathrm{CT})$, respectively. This floor-of-the-mouth human oropharyngeal SCC model is valuable for correlation of in vitro 
data with in vivo studies on tumor progression and to evaluate potential chemotherapeutic agents.

\section{Materials and methods}

Cell culture

UM-SCC-(1, 17A, 17B, 22A, 22B, 74A, 81B, 11A, 11B, $14 \mathrm{~A}, 5)$ and OSCC 3 head and neck SCC cell lines were cultured, as described previously $(8,9)$, in Dulbecco's modified Eagle's medium (DMEM, GIBCO, Grand Island, NY, USA) containing 10\% fetal bovine serum, penicillin $100 \mathrm{U} / \mathrm{ml}$, streptomycin $100 \mu \mathrm{g} / \mathrm{ml}$, and $50 \mu \mathrm{g} / \mathrm{ml} \mathrm{L}$-glutamine.

\section{Immunodeficient mouse tumor model}

All protocols for in vivo studies were approved by the University Committee on the Use and Care of Animals at the University of Michigan.

HNSCC cell lines were grown to $70 \%$ confluence prior to injection. Six-week-old athymic nude mice (NCr-nu/nu strain, NCI, Frederick, MD, USA, weight 18-25 gm) were anesthetized via intraperitoneal injection with $100 \mathrm{mg} / \mathrm{kg}$ ketamine and $10 \mathrm{mg} / \mathrm{kg}$ xylazine. SCC cells were suspended in $0.2 \mathrm{ml}$ of DMEM and injected submucosally in the floor of the mouth. UMSCC-11B cells were suspended in 0.02-0.2 ml DMEM prior to injection. The animals were killed and the primary tumor and adjacent tissues, lungs, and liver were harvested, rinsed in phosphate buffered saline (PBS) and fixed overnight in $10 \%$ buffered formalin. The tissue was paraffin-embedded, sectioned, and stained with hematoxylin and eosin. Samples containing bone were decalcified in $10 \%$ EDTA (pH 7.4) prior to sectioning.

\section{Matrigel technique}

UM-SCC-5 and UM-SCC-11A cells in DMEM, were chilled on ice, and suspended in an equal volume of Matrigel (BD Biosciences, San Jose, CA, USA) basement membrane matrix in the presence or absence of growth factors (EGF, bFGF, NGF, PDGF, IGF-1, TGF- $\beta$ ) at a final concentration of $1 \times 10^{5}-2 \times 10^{6}$ cells $0.1 \mathrm{ml}$, respectively. The cell/matrigel solution was chilled on ice prior to the floor-of-mouth injection.

\section{Live imaging - bioluminescence}

To assess SCC cell growth and tumor burden in vivo by bioluminescence, UM-SCC-11B $\left(2.5 \times 10^{5}\right)$ was stably co-transfected with the human proximal promoter containing $1974 \mathrm{bp}$ of $5^{\prime}$-flanking sequence of the receptor activator for nuclear factor $\kappa$ B ligand (RANKL) gene in reporter plasmid pGL3-Basic and pcDNA3.1, containing a mammalian selection marker to generate stably transfected clones (Invitrogen, Carlsbad, CA, USA). In vivo real-time RANKL promoter activity was assessed by bioluminescence following intraperitoneal injection of D-luciferin substrate at $40 \mathrm{mg} / \mathrm{ml}$ of luciferin substrate in PBS at a dose of $150 \mathrm{mg} / \mathrm{kg}$ body weight essentially, as described previously (10) using the Xenogen IVIS imaging system (Xenogen Corp., Alamenda, CA, USA) every 2 weeks over an 8-week period. Subsequently, animals were euthanized and the mandibles were disarticulated at the level of the condyle using a diamondseparating disk. Block sections were immersed directly in $10 \%$ buffered formalin for at least $72 \mathrm{~h}$.

\section{Micro-computed tomography}

Non-demineralized rat maxillae were scanned in $70 \%$ ethanol by a cone beam $\mu \mathrm{CT}$ system (GE Healthcare BioSciences). Each scan was reconstructed at a mesh size of $18 \times 18 \times 18 \mu \mathrm{m}$ and three-dimensional digitized images were generated for each specimen. Using GEHC MicroView software (version Viz +2.0 build $0029 \mathrm{GE}$ Healthcare BioSciences), the images were rotated into a standard orientation and threshold to distinguish between mineralized and non-mineralized tissue. For each specimen, a grayscale voxel value histogram was generated to determine an optimal threshold value. Loss of bone volume caused by local invasion of HNSCC into the mandible was assessed using three-dimensional isoform displays. After proper image orientation, the region of interest (ROI) was determined. The standard ROI (volumetric box of $250 \times 200 \times 200$ pixels) was used from the inferior alveolar foramen. After establishing the threshold at 1621, the bone volume fraction was calculated as the percent of bone within the ROI.

\section{Results}

Optimization of xenograft mouse model of human head and neck squamous cell carcinoma

The murine floor-of-mouth model for human oral cancer was optimized for xenografts using several SCC

Table 1 Summary of human HNSCC cells lines tested in murine floor-of-mouth model

\begin{tabular}{|c|c|c|c|}
\hline Cell lines & $\begin{array}{l}\text { Cell concentration } \\
\quad\left(\times 10^{6} \text { cells }\right)\end{array}$ & $\begin{array}{l}\text { Positive takes } \\
\quad \text { (tumor) }\end{array}$ & $\begin{array}{c}\text { Failures } \\
\text { (no tumor) }\end{array}$ \\
\hline UM-SCC-1 & 1 & 2 & 0 \\
\hline UM-SCC-17A & 2 & 4 & 0 \\
\hline \multirow[t]{3}{*}{ UM-SCC-17B } & 0.5 & 6 & 1 \\
\hline & 1 & 17 & 2 \\
\hline & 2 & 5 & 1 \\
\hline UM-SCC-22A & 1 & 14 & 1 \\
\hline \multirow[t]{2}{*}{ UM-SCC-22B } & 0.5 & 4 & 0 \\
\hline & 1 & 10 & 0 \\
\hline UM-SCC-74A & 2 & 6 & 0 \\
\hline UM-SCC-81B & 1 & 6 & 1 \\
\hline \multirow[t]{6}{*}{ UM-SCC-11B } & 0.05 & 0 & 2 \\
\hline & 0.25 & 6 & 0 \\
\hline & 0.5 & 3 & 1 \\
\hline & 1 & 4 & 1 \\
\hline & 3 & 2 & 0 \\
\hline & 5 & 3 & 0 \\
\hline \multirow[t]{2}{*}{ OSCC3 } & 1 & 2 & 0 \\
\hline & 2 & 2 & 0 \\
\hline \multirow{2}{*}{ UM-SCC-14A } & 1 & 0 & 2 \\
\hline & 2 & 0 & 2 \\
\hline \multirow[t]{3}{*}{$\mathrm{UM}-\mathrm{SCC}-5^{\mathrm{a}}$} & 0.5 & 2 & 0 \\
\hline & 1 & 2 & 0 \\
\hline & 2 & 2 & 0 \\
\hline \multirow[t]{2}{*}{ UM-SCC-11A } & 0.1 & 2 & 0 \\
\hline & 0.5 & 2 & 0 \\
\hline
\end{tabular}

${ }^{\mathrm{a}}$ Cell lines requiring matrigel. 
cell lines, UM-SCC-(11A, 11B, 17A, 17B, 1, 5, 74A, $81 \mathrm{~B}, 22 \mathrm{~A}, 22 \mathrm{~B})$ and OSCC3. The tumor cell dose, number of positive takes, and number of failures are shown in Table 1. Tumor cell dose was optimized for each cell line to produce $4-5 \mathrm{~mm}$ tumors, corresponding to a palpable tumor volume of $35-60 \mathrm{~mm}^{3}$, within $\sim 2-$ 4 weeks post-injection. UM-SCC-17B xenografts exhibited morphologic and cytologic features compatible with poorly differentiated HNSCC consistent with the nature of the recurrent tumor from which this cell line was developed $(9,10)$. UM-SCC-17B (Fig. 1A, B) exhibited islands and sheets of malignant epithelial cells exhibiting nuclear hyperchromatism, nuclear pleomorphism, and numerous atypical mitoses (1B, arrows). Similar histopathologic features were observed with other HNSCC cell lines (UM-SCC-22A, UM-SCC-74A, OSCC3). Additionally, microscopic examination of the liver (Fig. 1C, D) and lung (Fig. 1E) showed atypical mitoses (arrows) and nuclear pleomorphism (arrowheads) at these sites, but this was not a predictable finding.
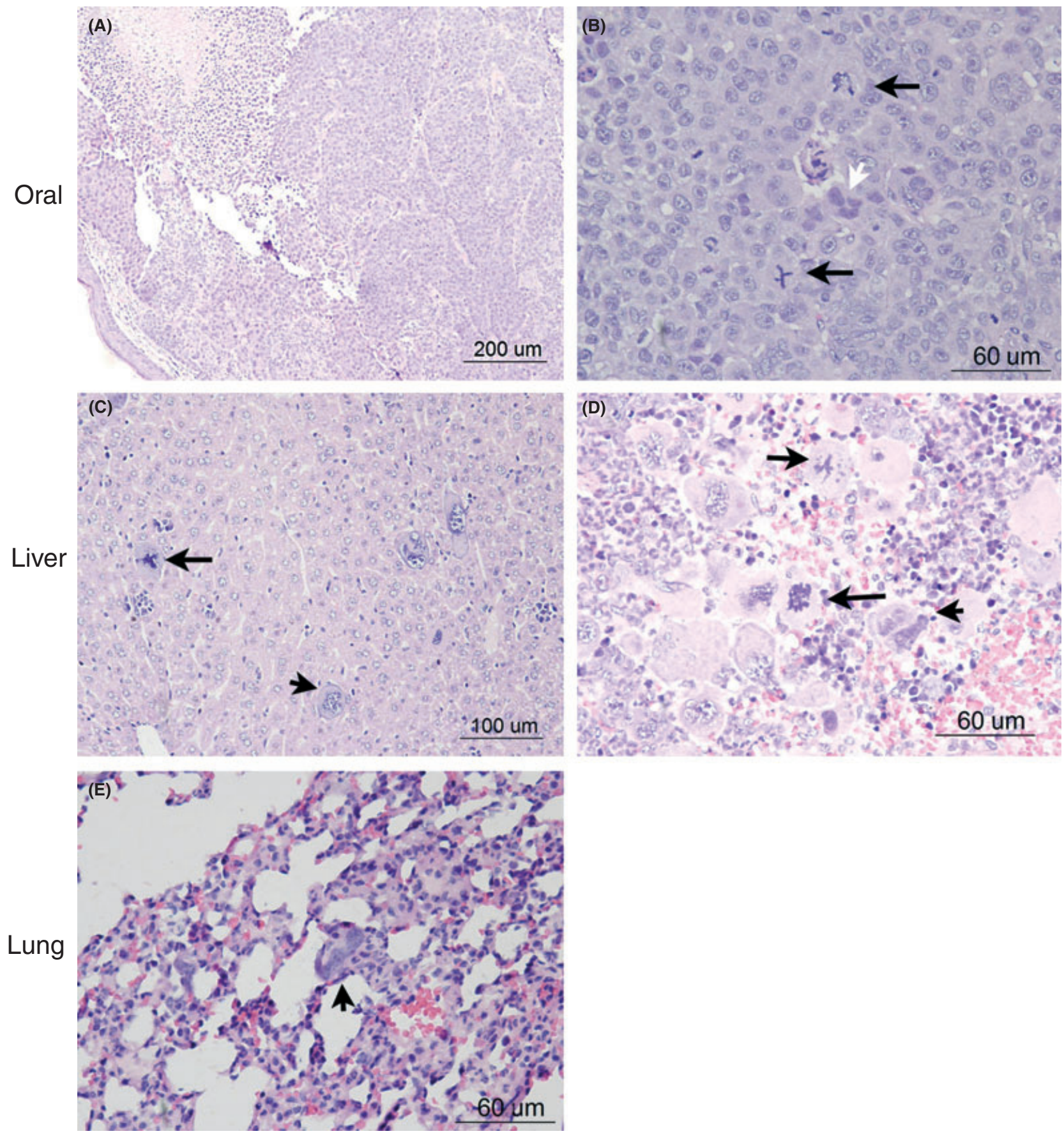

Figure 1 Tumors are produced in the floor of the mouth, 3 weeks post-injection of UM-SCC-17B cells. (A, B) Primary oral tumor. (C, D) Metastatic tumor in the liver. (E) Occasional neoplastic cells were observed in the lung. Pleomorphic neoplastic cells are observed in the liver (C, D) and lung (E) (arrowheads). A typical mitoses are indicated by arrows. 
Matrigel facilitates tumorigenesis of squamous cell carcinoma cells

Despite the large panel of HNSCC cells that were tumorigenic in mice, other cell lines such as UM-SCC5 and UM-SCC-11A did not grow in the floor of mouth (up to $5 \times 10^{6}$ cells). As these SCC cell lines vary in genetic mutations and chemotherapy resistance (11), a more robust murine model of HNSCC is essential. Hence, these cell lines were suspended in a basement membrane matrix. The matrix forms a gel at body temperature, thereby facilitating localization of tumor cells. UM-SCC-5 and UM-SCC-11A (0.5$2 \times 10^{6}$ and $0.1-0.5 \times 10^{6}$ cells, respectively) induced tumors in $\sim 3-4$ weeks following injection. UM-SCC-5 tumors exhibited invasion of sheets and nests of poorly differentiated tumor cells into the surrounding tissues (Fig. 2A) and the lung (Fig. 2B). The histology is consistent with the original tumor pathology report that described the surgical specimen used to establish this cell line as 'poorly differentiated squamous cell carcinoma'. Tumor islands (Fig. 2C, D, arrows) of UM-SCC-11A exhibited keratin pearl formation, nuclear hyperchromatism and pleomorphism and invaded skeletal muscle (Fig. 2C, arrowheads) and bone (Fig. 2D). Tumor islands invading the surrounding

structures were also observed with UM-SCC-5A (data not shown). Thus, the Matrigel modification technique can be used to support in vivo tumor development with cell lines that typically do not grow with standard procedures.

\section{Live imaging of tumor growth}

The proximal promoter of the human RANKL gene was used to induce expression of a luciferase reporter to follow in vivo expression of luciferase in implanted UMSCC-11B cells. Thus, reporter activity could be used to monitor in vivo expression and tumor burden. Using this approach, UM-SCC-11B-RANKL-Luc cells were injected into floor of the mouth $\left(0.25 \times 10^{6}\right.$ cells; $\left.n=6\right)$ of athymic (nu/nu SCID) mice. To identify tumor growth, radiographs of the head and neck region at 2, 6, and 8 weeks post-tumor injection were taken (Fig. 3A). Radiographic changes, including osteolytic changes and soft tissue tumor growth, were not identified until 6 weeks after UM-SCC-11B-RANKL-Luc cell injection. The changes were readily evident at 8 weeks. Tumor was easily identifiable as early as 2 weeks by visual examination, palpation, and using bioluminescence imaging (BLI) and increased every 2 weeks until killing at 8 weeks (Fig. 3B). BLI was easily quantifiable

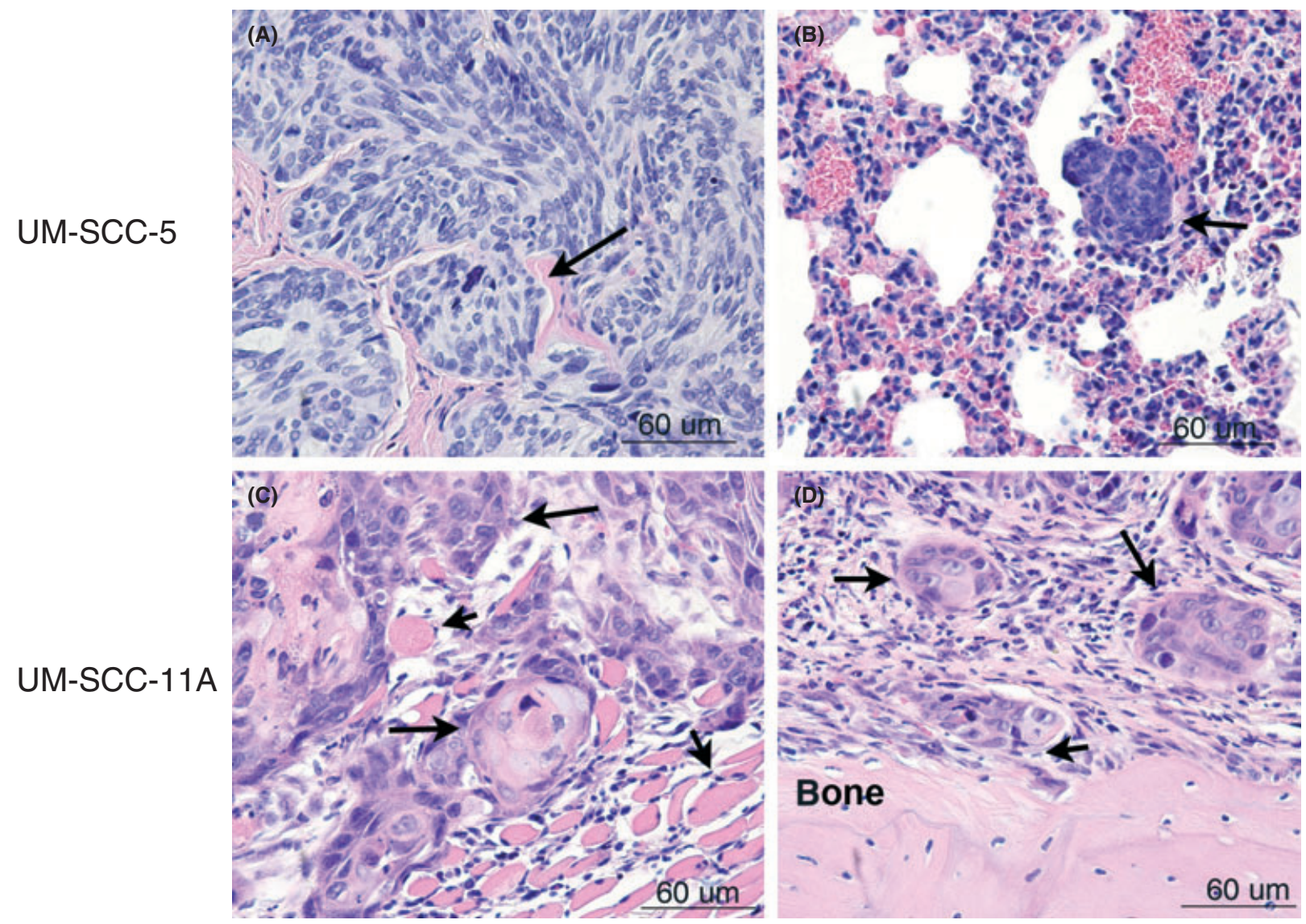

Figure 2 Matrigel facilitates tumor induction with cell lines that are otherwise non-tumorigenic. UM-SCC-5 $\left(1 \times 10^{6}\right)$ and UM-SCC- 11 A $\left(0.1 \times 10^{6}\right)$ cells were suspended in Matrigel and injected into the floor of the mouth. Primary (A) and metastatic (B) tumors were identified with UM-SCC-5. UM-SCC-11A islands (arrows) exhibited skeletal muscle (C, arrowheads) and bone invasion (D). 

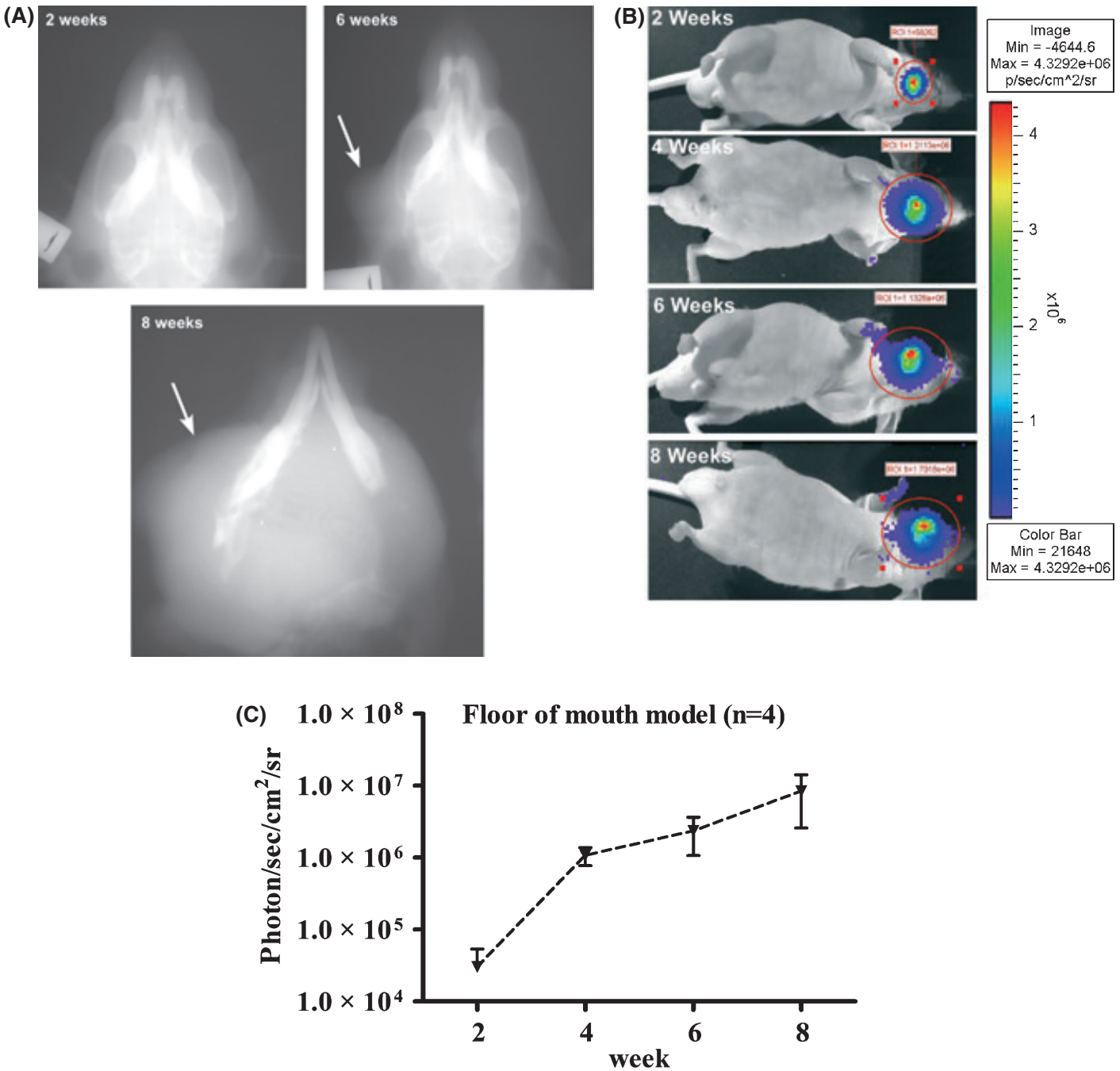

Figure 3 Tumor growth can be monitored by Faxitron X-ray and bioluminescence. UM-SCC-11B-RANKL-Luc reporter cells $\left(0.25 \times 10^{6}\right)$ were injected into the floor of the mouth and tumor growth was monitored over an 8-week period. (A) Faxitron X-rays from 2-, 6-, and 8-week time points indicate tumor growth (see arrow) and loss of mineralization consistent with tumor invasion of mandible. (B) Representative bioluminescence images of mice over the 2- to 8-week time frame. The same mouse is shown over this time period. Color bar indicates luminescence intensity from low (blue) to high (red). (C) Graphical representation of photon counts of bioluminescence data obtained from three animals over 8 -week time period. Luminescence emitted from each mouse was integrated over 1-min exposure starting 10 min after D-luciferin injection.

as shown in Fig. 3C. These data indicate that BLI can be used to non-invasively measure SCC tumor growth in the floor of the mouth.

\section{Micro-computed tomography permits three-dimensional visualization of tumor-bone interaction}

Following killing, non-decalcified specimens were scanned by $\mu \mathrm{CT}$. Three-dimensional isoform images (Fig. 4) indicate extensive bone destruction (lower panel) compared with non-tumor control specimen (upper panel). In addition, note that molar teeth appear to be lost because of extensive bone loss in this model.

Histology confirms the presence of local invasion of oral cancer in the floor-of-mouth model. The mandible and adjacent tissues were harvested and decalcified. Sections through the tongue, floor of mouth, and mandible confirmed the presence of an aggressive malignant epithelial infiltrate (Fig. 5A, B) that had invaded bone (Fig. 5D, C, F, arrows) and extended to the tooth surface (Fig. 5D-F). As expected, SCC cells were present in the floor of the mouth and had invaded adjacent structures (Fig. 5A, arrow), underscoring the importance of this model in replicating the human disease.

\section{Discussion}

Any investigation of the etiology and pathogenesis of a disease requires in vivo models that recapitulate the disease. Furthermore, the development of new drug and therapeutic regimens to combat head and neck cancer requires pre-clinical testing using murine models. Existing tumor models often require injection of tumor cells into the flank or nape of mice, and therefore do not accurately recapitulate the vasculature, mucosa, musculature, and nerves of the upper aerodigestive tract. Head and neck cancer presents specific challenges because of 


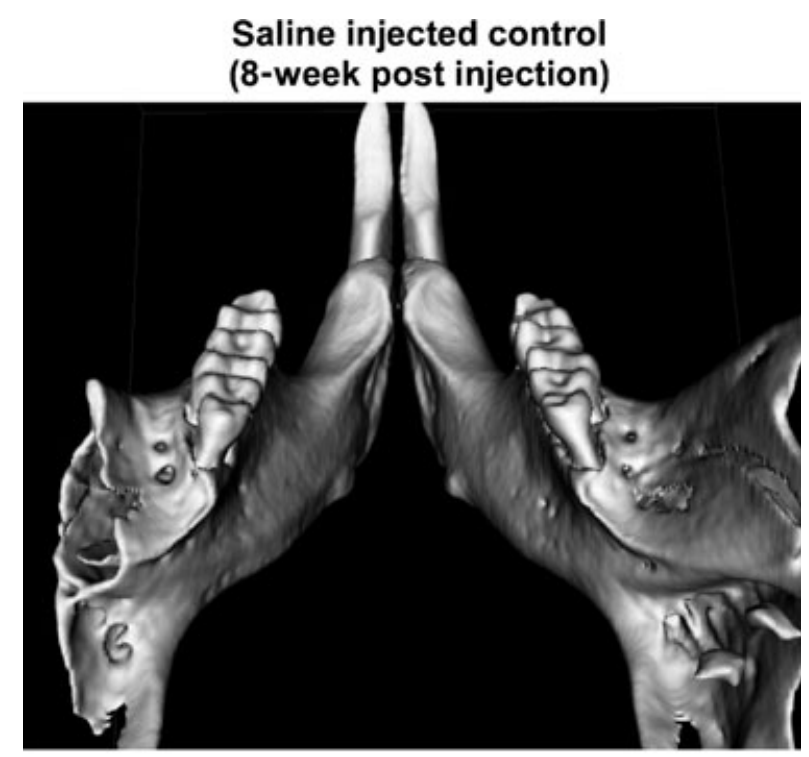

UMSCC-11B injected (8-week post injection)

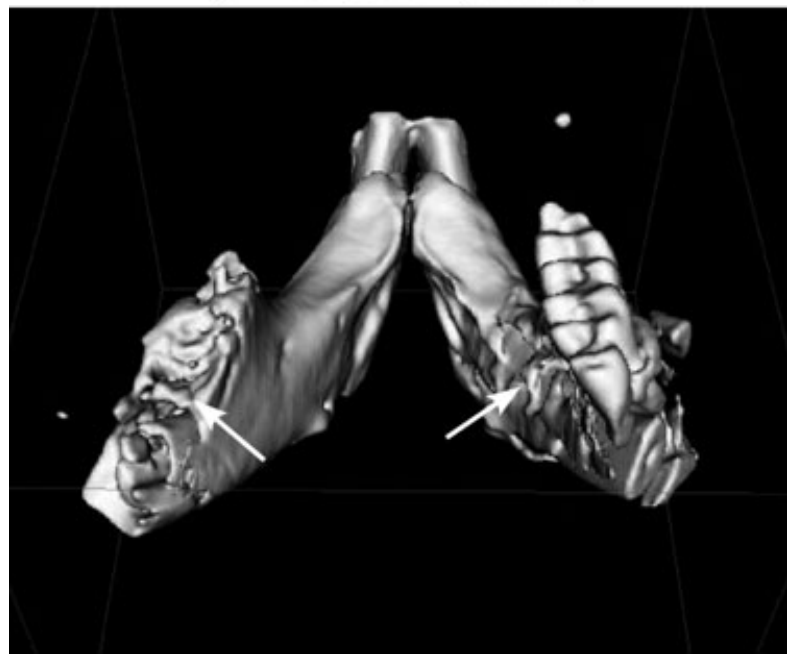

Figure 4 Micro-CT shows local tumor invasion in the mandible. UM-SCC-11B-RANKL-Luc reporter cells $\left(0.25 \times 10^{6}\right)$ were injected into the floor of the mouth. Eight-week post-injection mice were killed for $\mu \mathrm{CT}$ imaging. Representative reformatted $\mu \mathrm{CT}$ isoform displays from saline-injected (upper panel) and tumor-injected (lower panel) mice. Arrows indicate tooth and bone loss in tumor-injected animals.

locoregional aggressiveness associated with direct extension into cervical structures, and thus requires a model that more closely recapitulates the natural progression of the disease $(4,7)$. This point is illustrated quite nicely by Kawashiri and colleagues, who injected 152 nude mice intra-orally and subcutaneously on the back with two established oral SCC cell lines, OSC-19 and OSC-20 (12). They described the back tumors, histologically and on gross dissection, as having a more expansive but poorly invasive growth pattern. In contrast, tumors derived from SCC cells implanted in the floor of the mouth were histologically similar to the original tumors and were significantly more invasive in surrounding tissues (12). In the present study, we observed highly aggressive HNSCCs, similar in histology to the human HNSCCs from which they were established.

Despite the apparent advantages of a floor-of-mouth approach to study head and neck cancer, a number of significant challenges existed with previous models. First, techniques using human SCC cells required large numbers of cells (typically $2.0 \times 10^{6}$ cells) to obtain a 'critical mass' of cells sufficient to produce tumors (7). This large number of cells, however, sometimes results in very large tumors, and killing of the animals at 2 weeks post-injection becomes necessary. Therefore, these models are precluded from being used for longterm drug efficacy studies. Because previous laboratories used relatively large volumes of Hanks buffered saline for the cell suspensions, it is reasonable to speculate that large numbers of cells were necessary because of inevitable leakage and loss of cells. For example, Simon and colleagues reported relatively large injection volumes into the floor of the mouth, at a depth superficial to the mylohyoid muscle (7). In contrast, we were able to obtain tumors with as few as $0.25 \times 10^{6}$ human SCC cells (Fig. 5) suspended in a much smaller volume of $0.2 \mathrm{ml}$ of plain DMEM, and were able to follow these animals 8 weeks after injection. Thus, we developed a strategy whereby we could predictably grow tumors in the floor of the mouth of nude mice for a long period of time and with low tumor cell suspension volume and tumor cell dose. Furthermore, using bioluminescence and $\mu \mathrm{CT}$, tumor growth, and bone invasion can be monitored in vivo.

Our optimized technique resulted in successful tumor growth using 10 different human SCC cell lines; UMSCC-(1, 11B, 17A, 17B, 74A, 81B, 22A, 22B) and OSCC 3 cells. Despite this success, however, there were some cells that did not produce tumors using our optimized or standard injection techniques. The inability to grow certain cell lines using the model significantly limits its utility for those investigators that must use a particular cell line for reasons specific to their research. For example, the UM-SCC-5 (parental) and UM-SCC5PT (cisplatin resistant) cell lines have been used to study cisplatin resistance in vitro(11), and could also serve as an effective model system to study chemotherapy effectiveness and synergy in vivo. The UM-SCC-5 or UM-SCC-11A cell lines did not grow in the floor-ofmouth model using standard procedures. However, by embedding $0.5-2 \times 10^{6}$ or $0.1-0.5 \times 10^{6}$ cells, respectively, in Matrigel, we were able to obtain palpable tumors at 2-4 weeks post-injection.

The Matrigel preparation mimics natural basement membrane in that it contains type IV collagen and laminin, as well as proteases (MMP-2, MMP-9, plasminogen activator) (13). It may also contain growth factors (EGF, bFGF, NGF, PDGF, IGF-1, TGF- $\beta$ ), sulfated macromolecules, and glycosylated molecules (13). The exact mechanism by which Matrigel promotes SCC cell growth is not known; however, it is plausible that the angiogenic and growth factors embedded in the Matrigel provides a microenvironment that is conducive to the establishment of solid tumors $(13,14)$. Additionally, cells embedded in Matrigel become 

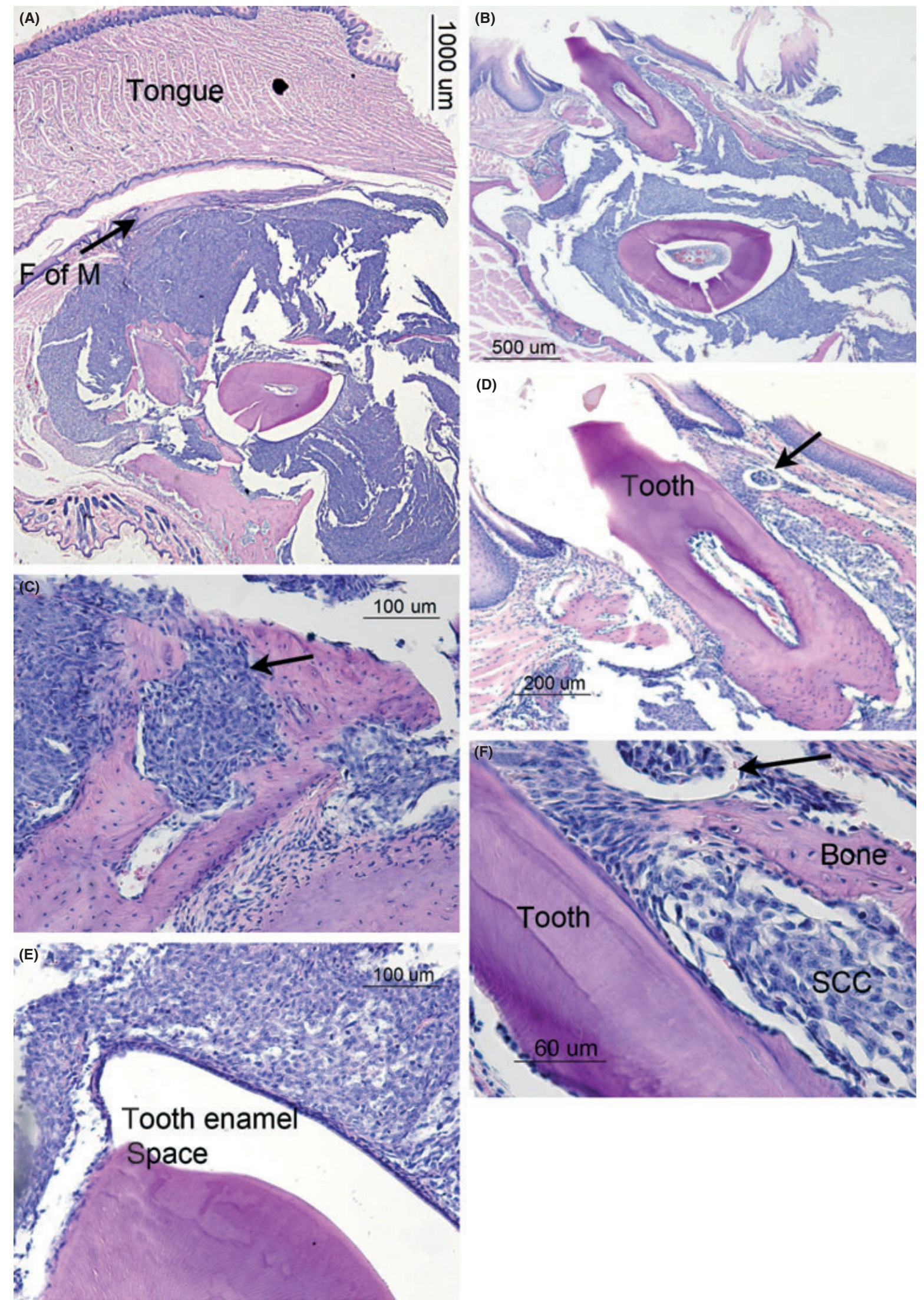

Figure 5 Tumor growth was apparent in the floor of the mouth (A, B; F of M). The tumor islands destroyed bone (B, C, D, F) and extended to the enamel surface $(\mathrm{E})$. 
polarized and also remain in closer proximity to each other than in a buffer solution, which could also serve to increase the locoregional level of angiogenic and growth factors as a result of autocrine and paracrine release (13).

Receptor activator for nuclear factor $\kappa \mathrm{B}$ ligand is an essential cytokine required for full osteoclast maturation and activation $(15,16)$. Initial studies indicated that stably transfected UM-SCC-11B cells harboring this plasmid express high levels of luciferase reporter in vitro. Hence, these cells were used to monitor tumor growth by bioluminescence in vivo. Recent evidence suggest tumor invasion and osteolytic activity requires RANKL (15), but this question is still in debate with oral SCC (17) and was not investigated in the present study. In vitro findings suggest that all SCC cell lines express RANKL (data not shown). In the present study, bone invasion was observed even in the absence of exogenous RANKL (Fig. 2D).

As a result of significant modifications, we have made this floor-of-the-mouth human oropharyngeal cancer model more effective for in vivo studies on tumor progression and for the evaluation of potential chemotherapeutic agents (18).

\section{References}

1. Todd R, Donoff RB, Wong DT. The molecular biology of oral carcinogenesis: toward a tumor progression model. J Oral Maxillofac Surg 1997; 55: 613-23 discussion 623-15.

2. Holmes JD, Dierks EJ, Homer LD, Potter BE. Is detection of oral and oropharyngeal squamous cancer by a dental health care provider associated with a lower stage at diagnosis? J Oral Maxillofac Surg 2003; 61: 285-91.

3. O’Malley BW Jr, Cope KA, Johnson CS, Schwartz MR. A new immunocompetent murine model for oral cancer. Arch Otolaryngol Head Neck Surg 1997; 123: 20-4.

4. Sales E. Growth and histopathology of human head and neck squamous cell carcinoma implanted intraorally in nude mice. Otolaryngol Head Neck Surg 1991; 104: 351-7.

5. Kawashiri S, Kumagai S, Kojima K, Harada H, Nakagawa K, Yamamoto E. Reproduction of occult metastasis of head and neck cancer in nude mice. Clin Exp Metastasis 1999; 17: 277-82.

6. Frijhoff AF, Conti CJ, Senderowicz AM. Advances in molecular carcinogenesis: current and future use of mouse models to screen and validate molecularly targeted anticancer drugs. Mol Carcinog 2004; 39: 183-94.

7. Simon C, Nemechek AJ, Boyd D, et al. An orthotopic floor-of-mouth cancer model allows quantification of tumor invasion. Laryngoscope 1998; 108: 1686-91.

8. Carey TE. Head and neck tumor lines. In: Hay R, et al. eds. Atlas of human tumor cell lines. Orlando, Florida: Academic Press, Inc., Harcourt Brace Jovanovich, Publishers, 1994; 79-120.

9. Lansford C. Head and neck cancers. In: Masters J ed. Human cell culture Vol 2, cancer cell lines Part 2. The Netherlands: Kluwer Academic Press, 2000; 185-255.

10. Rehemtulla A, Stegman LD, Cardozo SJ, et al. Rapid and quantitative assessment of cancer treatment response using in vivo bioluminescence imaging. Neoplasia 2000; 2: 491-5.

11. Bauer JA, Trask DK, Kumar B, et al. Reversal of cisplatin resistance with a $\mathrm{BH} 3$ mimetic, (-)-gossypol, in head and neck cancer cells: role of wild-type p53 and Bcl-xL. Mol Cancer Ther 2005; 4: 1096-104.

12. Kawashiri S, et al. Development of a new invasion and metastasis model of human oral squamous cell carcinomas. Eur J Cancer B Oral Oncol 1995; 31B: 216-21.

13. Kleinman HK, Martin GR. Matrigel: basement membrane matrix with biological activity. Semin Cancer Biol 2005; 5: 378-86.

14. Morales TI. Transforming growth factor-beta and insulinlike growth factor-1 restore proteoglycan metabolism of bovine articular cartilage after depletion by retinoic acid. Arch Biochem Biophys 1994; 315: 190-8.

15. Udagawa N. Mechanisms involved in bone resorption. Biogerontology 2002; 3: 79-83.

16. Khosla S. Minireview: the OPG/RANKL/RANK system. Endocrinology 2001; 142: 5050-55.

17. Tada T, Jimi E, Okamoto M, Ozeki S, Okabe K. Oral squamous cell carcinoma cells induce osteoclast differentiation by suppression of osteoprotegerin expression in osteoblasts. Int $J$ Cancer 2005; 116: 253-62.

18. Wolter KG, Wang SJ, Henson BS, et al. (-)-gossypol inhibits growth and promotes apoptosis of human head and neck squamous cell carcinoma in vivo. Neoplasia 2006; 8: $163-72$. 